\title{
Généricité / spécificité d'un concept: La transposition didactique en Sciences économiques et sociales
}

\section{Christine Dollo}

Le "projet fondateur» des Sciences économiques et sociales semblait à l'origine incompatible avec la prise en compte, par la didactique de la discipline, de la théorie de la transposition didactique. L'objectif de cet article est cependant de mettre en évidence la portée heuristique du concept inauguré par $M$. Verret puis théorisé par $Y$. Chevallard, pour les SES.

Mais on notera que, même si le concept utilisé initialement en didactique des mathématiques ou des Sciences physiques est tout à fait opératoire pour les SES, il est possible de mettre en évidence certains traits spécifiques de la transposition didactique en SES, liés aux caractéristiques propres de cette discipline scolaire. Ce travail sinscrit donc dans une perspective comparatiste.

\section{Introduction}

Lorsque la discipline scolaire Sciences économiques et sociales (SES) se crée en 1966-67, les textes originaux définissent ce que certains nommeront plus tard le "projet fondateur» 1 , qui présente trois caractéristiques majeures:

- Il s'agit d'une part de conduire les élèves à l'intelligence des économies et des sociétés contemporaines, c'est-à-dire à l'étude d'un certain nombre de questions, jusque-là absentes de l'enseignement secondaire général.

- Le projet s'inscrit d'autre part dans une perspective de l'unité des Sciences sociales, en soulignant que le découpage en disciplines étanches ne permet pas de rendre compte du social dans sa complexité. Il s'agit alors de partir de l'étude d'un certain nombre d' "objets» (la famille, la monnaie, le chômage) et de mobiliser de façon intégrée l'apport des diverses sciences sociales, en construisant une discipline scolaire ad hoc:

A la question 'quelle économie enseigner dans le second cycle du second degré de l'enseignement de l'enseignement général?' Marcel Roncayolo ${ }^{2}$ répond en 'fabricant' une discipline véritablement nouvelle en ce sens qu'elle ne constitue pas un simple 'modèle réduit' de ce qui se fait dans l'enseignement supérieur (Brémond \& Lanta, 1995, p. 64). 
- Enfin, ce projet intègre une approche pédagogique novatrice qui emprunte ses différents éléments aux divers courants pédagogiques qui critiquent l'école traditionnelle, notamment la mémorisation mécanique et le cours magistral. Dans cette nouvelle discipline scolaire, on insiste sur la volonté de favoriser l'activité des élèves, le travail sur documents, la pratique des enquêtes. (Beitone, Decugis, Dollo \& Rodrigues, 2004, p. 12)

Les textes officiels initiaux prônent une pédagogie active devant permettre à l'élève de "participer à l'élaboration de son propre savoir». On met l'accent sur «l'observation directe», le «concret», la «description». Les théories ne sont pas évoquées, tout au plus les «mécanismes» que l'on propose de n'étudier que "progressivement, à partir d'exemples concrets. On préconise de mettre les élèves en activité mais on ne fait aucune référence à leurs conceptions ou représentations sociales du monde qui les entoure.

Ce projet fondateur mêle ainsi étroitement une finalité (se confronter aux questions de société), des options épistémologiques (la possibilité de bâtir une discipline scolaire $a d h o c$ ) et un positionnement pédagogique fort (inductiviste). Dans sa définition même, ce projet semblait à l'origine incompatible avec la prise en compte, par la didactique de la discipline, de la théorie de la transposition didactique. Aussi ce concept est-il assez vivement rejeté par certains des premiers travaux de didactique des SES ainsi que nous le verrons dans une première partie de cet article.

Pourtant, pour sauvegarder la finalité (permettre aux élèves d'accéder à l'intelligence des sociétés contemporaines), il faut accepter de remettre en discussion les deux autres composantes du "projet fondateur» (une "approche intégrée du social» et une démarche inductive). De fait, des évolutions récentes de la discipline - dans les programmes et les instructions officielles - vont à la fois dans le sens d'une prise en compte des représentations sociales des élèves et d'une introduction d'éléments de théorisation dans les curricula. Les SES tendent à devenir une discipline scolaire basée sur de solides savoirs scientifiques de référence, s'appuyant sur les conceptions des élèves pour aider ceux-ci à élaborer une véritable connaissance des problèmes économiques et sociaux contemporains. L'objectif de la deuxième partie de cet article est alors d'insister sur le fait que cette discipline scolaire constitue bien aujourd'hui un champ d'application de la théorie de la transposition didactique de Chevallard (1985/1991).

Dans la troisième et dernière partie enfin, nous présentons quelques éléments relevant d'après nous d'une certaine "spécificité» de la transposition didactique en SES. Ils concernent notamment la dépersonnalisation du savoir et un certain renversement du processus classique de la transposition didactique sous l'influence des outils d'évaluation.

Ce travail s'inscrit donc dans une perspective comparatiste, inaugurée notamment par Mercier, Schubauer-Leoni et Sensevy (2002). 


\section{La transposition didactique: un concept d'emblée contesté par la discipline scolaire Sciences économiques et sociales}

\section{Origine d'un concept}

En 1974, Michel Verret définit la didactique comme «la transmission d'un savoir acquis. Transmission de ceux qui savent à ceux qui ne savent pas. De ceux qui ont appris à ceux qui apprennent» (Verret, 1975, p. 139) et l'idée selon laquelle on ne peut pas enseigner un savoir, tel qu'il est produit dans les communautés savantes, sans transposition, se diffuse alors à travers la communauté des chercheurs en éducation.

C'est ensuite au début des années 80 qu'Yves Chevallard, didacticien des mathématiques, reprend à son compte cette idée et théorise le processus de transposition didactique en mathématiques ${ }^{3}$. Dans son ouvrage de 1985 , Chevallard tient à réhabiliter le savoir et définit le concept de transposition didactique comme le passage du savoir "savant» ${ }^{4}$ au savoir enseigné et renvoie donc à l'obligatoire distance qui les sépare. Il précise ensuite plus récemment que, de façon générale, la théorie de la transposition didactique stipule une certaine «migration» de savoirs d'une sphère institutionnelle à une autre et tente de déterminer au mieux les caractéristiques de cette migration. Dans l'institution scolaire par exemple, "vivent» un certain nombre de savoirs que les «sujets» (les élèves) de cette institution doivent apprendre. Mais ces savoirs ont trouvé leur genèse dans une autre Institution que l'institution scolaire. La présence de ces savoirs dans l'école, et plus précisément dans le système d'enseignement associé à cette institution scolaire suppose donc qu'il y ait eu une «transposition didactique». Pour Chevallard, le «travail transpositif apparaît ainsi essentiel dans le fonctionnement et la survie des institutions» (Chevallard, 1996, p. 194). Le problème essentiel pour la société est alors «celui de l'identification et du choix des savoirs que la société voudra voir enseignés dans son école» (p. 196). Toute institution est ainsi "affrontée au problème du 'choix' des savoirs qu' elle regardera comme pertinents pour la formation de ses sujets». (p. 196)

Cinq éléments essentiels se dégagent de cette construction théorique:

- L'importance accordée au savoir dans le fonctionnement des systèmes d'enseignement.

- L'importance accordée au contrôle que le système social (via la noosphère) exerce sur les systèmes didactiques: «nul savoir enseigné ne saurait s'autoriser de lui-même» (Chevallard, 1994, p. 146).

- L'importance accordée à la dimension institutionnelle des questions relatives à l'enseignement.

- L'importance accordée à la dynamique des savoirs enseignés. Ceux-ci sont frappés d'obsolescence, leur contenu doit évoluer afin de gérer leur distance aux savoirs de référence. Il existe donc des crises périodiques et des mouvements de réforme qui adaptent les savoirs à enseigner à la fois en fonction de 
la dynamique des savoirs de référence et de la dynamique sociale.

- L'importance accordée à la mise en texte du savoir et à la place déterminante de l'enseignant dans la transposition: «le texte du savoir enseigné n'est [donc] pas écrit dans les ouvrages où le savoir s'expose mais il appartient aux systèmes didactiques eux-mêmes.» (Mercier, 2002, p. 143). Selon Mercier ainsi, le savoir est construit, montré et pratiqué «dans un parcours que le professeur dirige comme s'il donnait une lecture» (p. 143).

Si le concept de transposition didactique est aujourd'hui reconnu comme fondement des didactiques de nombreuses disciplines, Mercier note cependant que, même en mathématiques, "cet accord ne s'est pas fait sans que des réticences virulentes ne se soient manifestées ni sans que des débats virulents (...) se poursuivent» (Mercier, 2002, p. 137).

De tels débats existent également en SES.

Un vif débat dans les années quatre-vingt dix Au début des années quatre-vingt-dix, la référence à la théorisation de Chevallard fait l'objet de vifs débats traversant la discipline; deux positions s'y affrontent: la position «fondamentaliste» et la position des «refondateurs» (Legardez, 2001).

Les fondamentalistes revendiquent la fidélité au projet fondateur des SES: «seuls ceux qui rejettent l'objectif fondateur sont nos ennemis» (Combemale, 1995 , p. 6). Ces auteurs affirment ainsi clairement que trente après la naissance de la discipline, "la situation n'a pas changé: fondamentalement, il n’y a pas d'alternative au projet conçu en 1966, pas d'alternative à la pédagogie avec laquelle il forme un tout» (Brémond \& Lanta, 1995, p. 69). Du point de vue épistémologique tout d'abord, ils adoptent une posture rétive à la théorisation et privilégiant la description. Leur conception de l'apprentissage des élèves ensuite, les conduit à définir les SES par leur méthode plutôt que par leurs contenus de savoir. Quant aux objectifs d'apprentissage fixés aux élèves, ils portent davantage sur une certaine attitude intellectuelle et sur l'autonomie des élèves que sur des connaissances: les fondamentalistes évoquent notamment régulièrement les risques d'encyclopédisme. (Beitone et al., 2004, p. 19). Du point de vue institutionnel enfin, même si la légitimité de la discipline scolaire est essentiellement politique et éthique, (il s'agit de former le citoyen, d'éduquer l'esprit critique) on insiste dans le projet fondateur sur le travail des professeurs «qui produiraient un 'savoir intermédiaire' autonome vis-à-vis des savoirs savants, légitimé par son 'enseignabilité”» (p. 20).

A l'inverse, les refondateurs considèrent qu'il faut d'abord partir de la critique de l'empirisme et de l'induction pour «asseoir les SES sur une épistémologie plus proche de l'épistémologie savante» (Beitone et al., 2004, p. 20), qu'il faut ensuite travailler avec les élèves sur la base des apports des théories socioconstructivistes des apprentissages et qu'il faut enfin faire reposer «la légitimité des SES comme discipline scolaire sur la référence à des savoirs validés par des communautés scientifiques» (p. 20). 
Les principales critiques qui sont apportées par les fondamentalistes au modèle de la transposition didactique concernent la question de la référence à un savoir savant, le fait que «les Sciences économiques et sociales ne sont pas une propédeutique» (Brémond \& Lanta, 1995, p. 64) à l'enseignement supérieur et que le travail des enseignants ne peut se résumer à la réduction d'écarts aux savoirs savants.

\section{Savoir savant et lien avec L'Université}

Pour Hadjian, deux éléments conduisent à refuser le modèle de la transposition didactique:

- «L'enseignement secondaire ne peut consister en un processus de transmission du savoir savant» (Hadjian, 1994, p. 76); il avance ainsi l'argument selon lequel la légitimation d'une discipline scolaire passe avant tout par le repérage de tâches et la mise en œuvre d'un processus d'évaluation.

- «La rupture de la 'transposition didactique' n'est praticable ni par les élèves, ni par les professeurs» (p. 76): selon lui, ni les professeurs ni les élèves ne peuvent partir des savoirs universitaires, d'autant plus que l'écrasante majorité des élèves de lycée scolarisés dans la série ES ne poursuivra pas d'études dans les facultés de Sciences économiques. Les SES ont selon lui vocation à être un enseignement de culture générale et doivent privilégier l'étude de la réalité économique et sociale. Dans ce cadre, mieux vaudrait pratiquer des enquêtes de terrains, plutôt que de n'enseigner «que des théories sans donner les outils en matière de connaissances historiques et de description pour, ne disons pas valider mais confronter ces théories au réel» (p. 77). En ce qui concerne les enseignants, il lui paraît également démesuré d'exiger d'eux qu'ils soient au fait de l'actualité de la recherche.

Ainsi pour lui:

si la référence utilitariste à la domination de l'économique peut nous procurer quelque avantage à court terme en matière de 'crédibilité,', elle risque de nous conduire à long terme à nier la spécificité de notre enseignement, c'est-à-dire, au mieux, à décalquer le savoir universitaire en économie, au pire à naturaliser comme loi éternelle cette domination (Hadjian, 1994, p. 78).

De la même façon, Brémond et Lanta (1995) insistent sur la différence de nature existant entre les savoirs à enseigner aux élèves et les savoirs universitaires:

Il en résulte que présenter aux élèves un condensé des 'savoirs universitaires' est peut-être valorisant pour l'enseignant qui 'expose' ses connaissances mais le plus souvent inefficace du point de vue de l'acquisition de savoirs maîtrisés par l'élève (p. 50). 


\section{De la transposition didactique à la transformation des savoirs}

La critique de Chatel (1995a) est plus modérée. Elle souligne le fait que nous devons à Verret (1975), puis à Chevallard (1985/1991) d'avoir mis en évidence à quel point l'activité des enseignants dans leurs classes concerne les savoirs:

Par le concept de transposition didactique, ces auteurs nous montrent que l'enseignement rend nécessaire d'effectuer des choix au sein du savoir de référence, d'en modifier la forme et même la structure du fait de son mode d'exposition, de son découpage, etc., afin de l'adapter à la situation d'enseignement. Le savoir, apprêté pour être enseigné, ne peut plus sidentifier purement et simplement au savoir 'savant' qu'il prend pour origine et pour référence (Chatel, pp. 10 - 11).

Cependant, dans ce modèle théorique, les élèves sont pour elle des récepteurs plus que des acteurs et la transposition didactique est le résultat d'une action qui se passe pour l'essentiel hors de la classe, dans la noosphère: «la figure ternaire élève - enseignant - savoir [...] pose le savoir comme un corpus ou comme un texte avec lequel deux sortes d'acteurs, supposés invariants, entretiennent des rapports» (Chatel, 1995a, p. 12). Même si Chatel admet l'existence, à la fin des années quatre-vingt, d'un assez large accord sur «la référence de la discipline aux savoirs savants ou du moins sur leur dénomination universitaire» (Chatel, Caron, Fenet-Chalaye, Le Merrer, Pasquier, \& Simula, 1993, p. 168), elle indique que ce qu'elle remet en cause dans la théorie de la transposition didactique, c'est bien le fait que ce concept se réduirait à une simple mesure de l'écart entre savoirs enseignés et savoirs savants. C’est également ce que regrette Triby (1995) en soulignant qu' «une conception trop restrictive de la transposition didactique nous installe confortablement dans le couple savoir savant - savoir enseigné en 'oubliant' qu'il n'y a pas que les savoirs qui circulent entre l'enseignant et l'élève» (p. 123). Chatel (1995a) préfere ainsi utiliser le concept de «transformation du savoir», qui permet de montrer «que le savoir qui est produit dans la classe est le fruit de l'interaction élèves - professeur» (p. 266). Pour opérer la substitution, il faut alors se défaire de cette identification du savoir à un texte: «l'idée de transformation nous permet de nous dégager du problème de la référence et de mieux prendre en compte dans l'analyse l'enseignement - apprentissage en tant que processus» (Chatel, Caron, Le Merrer, Pasquier, Richet \& Simula, 1995, p. 10). Chatel refuse ainsi ce qu'elle considère comme une interprétation trop substantivée de la transposition «qui fait de celle-ci un écart entre deux textes, plus qu'une tension entre deux formes d'activité» (Chatel, 1999, p. 23). Au contraire selon elle, «l'enseignement ne peut se réduire à l'émission d'un texte, par le professeur, en application des prescriptions officielles, texte qui serait reçu tel quel par les élèves» (Chatel, 1995b, p. 31). 


\section{La transposition didactique: un concept opéra- toire pour les Sciences économiques et sociales}

Contrairement aux auteurs que nous venons de citer, nous considérons pour notre part que le schéma proposé par Chevallard (1985/1991) pour définir la transposition didactique ${ }^{5}$ au sens large (Objet de savoir ( objet à enseigner ( objet d'enseignement) est tout à fait opératoire pour les SES. Par exemple, lorsque le programme de Seconde en SES prévoit l'étude du concept de chômage, il désigne ce concept comme objet à enseigner parce qu'il a été repéré par la noosphère comme un objet de savoir pertinent. Il va falloir ensuite un travail très important (auteurs de manuels, publications pédagogiques, corps d'inspection, associations professionnelles, instituts de formation des maîtres, enseignants, etc.) pour transformer cet objet de savoir en objet d'enseignement. Il faut pour cela sélectionner dans l'immense littérature sur le chômage des références jugées pertinentes (et d'abord une ou plusieurs définitions), concevoir des activités pédagogiques, des documents destinés aux élèves, ainsi que des instruments d'évaluation.

\section{Référence savante, "savoirs» et "pratiques»}

La question des savoirs savants est généralement considérée comme la question sensible pour les détracteurs de la théorie de la transposition didactique. Elle se traduit souvent par l'affirmation de la nécessité de partir des faits et des pratiques des élèves et par le refus de la référence savante. Pourtant, si les SES veulent participer à la formation de citoyens éclairés, elles doivent doter les élèves d'outils conceptuels et de connaissances scientifiques leur permettant d'accéder aux débats économiques et sociaux qui traversent notre société.

Lorsque les programmes prévoient l'enseignement de la monnaie, il ne s'agit pas d'enseigner la bonne manière de gérer son budget, ou la pratique de gestion de sa clientèle par le banquier. Les programmes de SES ont pour but d'expliquer aux élèves quelle est la nature de la monnaie, quels sont les mécanismes et les acteurs partie prenante dans le jeu complexe de la création de monnaie. Il ne s'agit pas non plus de partir de la pratique quotidienne des élèves consistant par exemple à régler leurs achats grâce à de la monnaie fiduciaire ou en utilisant un chèque ou une carte de crédit ${ }^{6}$. Car en effet, partir des pratiques des élèves, sur ce point précis du programme, pourrait constituer un obstacle à l'apprentissage dans la mesure où, par exemple, l'essentiel de la monnaie en circulation dans une économie est une monnaie scripturale, non «manipulée» au quotidien par les élèves. En cela, nous revendiquons l'héritage de Bachelard (1983), lorsqu'il écrit que «l'observation première est toujours un premier obstacle pour la culture scientifique. En effet, toute observation première [...] est pittoresque, concrète, naturelle, facile. Il n'y a qu'à la décrire et à s'émerveiller. On croît alors la comprendre» (p. 19). 
Il en va de même pour un autre thème au programme de la Seconde, le chômage. Dans les agences locales de l'Agence Nationale pour l'Emploi (ANPE), des formations sont assurées auprès des chômeurs à la recherche d'un emploi: on leur apprend à consulter des petites annonces, à repérer les caractéristiques d'un emploi sur une annonce, à répondre à ces annonces, à remplir des formulaires. Les savoirs ainsi transmis portent explicitement sur des pratiques sociales et nous semblent ainsi assez clairement relever de ce que Johsua (1998) appelle les «savoirs experts». Mais dans les programmes de SES, l'ANPE est abordée sous l'angle institutionnel et du point de vue de la construction sociale d'un indicateur de mesure. Il s'agit donc bien, à propos de ce thème, de montrer aux élèves comment la mesure de certains faits économiques repose sur des conventions, qui peuvent être différentes selon les institutions concernées, en l'occurrence ici l'ANPE ou l'INSEE, et qui sont elles-mêmes issues d'hypothèses théoriques concernant le mécanisme étudié.

Ainsi, pour analyser les problèmes que se pose actuellement la société, il n'est pas possible d'adopter une démarche purement descriptive comme le préconise le "projet fondateur». On ne peut en effet éviter la mise en relation avec des disciplines de référence, des «savoirs savants», pour construire des objets scientifiques comme celui du chômage.

En SES finalement, les références sont bien des savoirs et non des pratiques. Et de fait, les programmes, les manuels et les sujets de bac font référence à des questions, des concepts, des théories etc. qui relèvent de la référence à des «savoirs savants» et non d'une référence à des pratiques. Cela ne signifie pas pour autant que les faits doivent être absents de l'enseignement des SES, mais que les élèves ne peuvent accéder aux faits que s'ils disposent de la conceptualisation pertinente pour les aborder. Car la référence savante, dans l'optique de la transposition didactique, permet en effet, par la conceptualisation, de poser les bonnes questions relatives au monde économique et social contemporain:

L'esprit scientifique nous interdit d'avoir une opinion sur des questions que nous ne comprenons pas, sur des questions que nous ne savons pas formuler clairement. Avant tout, il faut savoir poser des problèmes. Et quoi quion dise, dans la vie scientifique, les problèmes ne se posent pas d'eux-mêmes. C'est précisément ce sens du problème qui donne la marque du véritable esprit scientifique. Pour un esprit scientifique, toute connaissance est une réponse à une question. S'il n'y a pas eu de question, il ne peut y avoir connaissance scientifique. Rien ne va de soi. Rien n'est donné. Tout est construit» (Bachelard, 1938, p.14).

\section{Unicité des savoirs savants, théorisation et transposition didactique}

Pour certains, l'absence d'unicité du savoir savant en SES rend impossible le recours au concept de transposition didactique. Certes, les SES ne s'appuient pas sur une seule discipline universitaire de référence. En outre, ces sciences de références sont elles-mêmes pluriparadigmatiques, ce qui rend en effet difficile la re- 
cherche d'un référent unique pour expliquer un phénomène économique et/ou social donné. Mais cela remet-il en cause l'idée d'un «apprêt didactique» du savoir avant de l'enseigner?

Bien plus, le caractère pluriparadigmatique des sciences de références est l'un des éléments essentiels qui doit conduire à interdire de partir des faits et de l'observation, mais bien d'objets de savoirs construits et repérés comme produits par une communauté savante. En ce qui concerne les explications du chômage, le savoir savant est protéiforme, et les grilles théoriques diverses. Et les savoirs à enseigner tels qu'ils apparaissent dans les programmes de la discipline, relèvent bien d'un processus de transposition didactique externe. Sur ce thème précis en effet, notre étude des manuels de Terminale ES (Dollo, 2001a) a montré qu'un nombre non négligeable d'entre eux font référence à des savoirs théoriques comme la «notion néoclassique de chômage volontaire», la «théorie des contrats implicites», la "théorie des salaires d'efficience», etc. Il ne s'agit pas là de connaissances qui vont être construites par la classe sur la base d'une observation des faits et d'une méthode inductive, mais bien de savoirs construits par la communauté savante, en réponse à des débats scientifiques, puis transposés dans l'institution scolaire pour y être enseignés parce qu'il permettent d'éclairer des problèmes économiques et sociaux contemporains.

En outre, nous avons pu montrer (Dollo \& Johsua, 2002) qu'on ne peut pas dépasser certaines conceptions contradictoires des élèves (à propos des explications du chômage par exemple), et mettre en évidence le caractère pluriparadigmatique de l'économie dès lors que l'on affirme que «l'enseignement trop précoce de modèles ou de schémas d'explication peut durcir de jeunes esprits et les rendre inaptes à entreprendre ultérieurement des études sérieuses de Sciences économiques et sociales» (Instructions Officielles, circulaire n ${ }^{\circ}$ IV $67-416 \mathrm{du}$ 12 octobre 1967). Pour partir des conceptions des élèves et construire avec eux des connaissances scientifiquement valides, la transposition d'objets de savoir académiques est non seulement possible mais absolument nécessaire.

En outre, un problème central est celui de la légitimité. Si les savoirs scolaires ne peuvent pas fonder leur légitimité sur leur rapport aux savoirs savants, sur quoi cette légitimité peut-elle reposer? Selon Chevallard (1994) en effet, il convient «que le savoir enseigné et le savoir qui lui sert, en quelque sorte, de caution épistémologique au regard de la Société, se ressemblent suffisamment» (p.146).

\section{La transposition didactique: un «processus vertical» descendant?}

Chatel (1999) reproche à la théorie de la transposition didactique le fait que les savoirs à enseigner viendraient "d'en haut», en insistant sur le fait que la réduction de la distance entre les savoirs scolaires et les savoirs savants ne doit pas être la seule variable à prendre en compte: «ce n'est pas la dynamique d'un rapprochement formel du texte du savoir à enseigner du texte savant qui permet de 
rendre compte de l'évolution du curriculum» (p. 28). Cette critique n'est pas véritablement fondée. En effet, Mercier souligne que l'on peut trouver, dans le travail de Chevallard, et notamment dans des articles à l'intention des professeurs, matière à

fonder aussi bien l'observation non prescriptive des enseignements effectifs (et les phénomènes relatifs au fonctionnement didactique qui sont observés) que l'ingénierie visant à produire certaines organisations de savoirs et à expérimenter leurs propriétés didactiques (enseignabilité, robustesse, justesse, pertinence) (Mercier, 2002, p. 140).

Par ailleurs, le fait d'insister sur l'importance du travail des enseignants dans leur classe, concernant la question des savoirs, n'est pas contradictoire avec la mise en œuvre d'un processus de transposition didactique. Nous pouvons ainsi, dans une certaine mesure, rejoindre Chatel en soulignant que les savoirs effectivement enseignés par le professeur font l'objet d'une réinterprétation du programme et des manuels (qui influence à l'évidence le contenu réellement enseigné) et que ce qui se passe entre un professeur et ses élèves est fondamental du point de vue de la construction des savoirs.

Plus fondamentalement encore, il est alors essentiel «d'entrer dans la classe» afin de mener une analyse du travail effectif du professeur et saisir la «réalité» du processus de «transposition interne» (Dollo \& Parayre, 2005). Cette affirmation nous permet de revenir sur l'importance de la textualisation du savoir pour comprendre l'enseignement dans ces différentes dimensions, et notamment du point de vue du rapport au temps didactique ou du point de vue des positions respectives du professeur et de l'élève dans la relation didactique (chronogénèse et topogénèse pour reprendre la typologie établie par Sensevy, Mercier \& SchubauerLeoni, 2000). La mise en texte du savoir ne signifie pas en effet que l'on se désintéresse de l'action effective (interactions professeur - élèves) dans laquelle le savoir à enseigner est mis en œuvre dans la classe pour conduire à des apprentissages. La transposition didactique permet de garantir la légitimité des objets de savoirs introduits dans la classe par le professeur.

Même si, comme nous venons de le montrer, les SES relèvent bien, du point de vue didactique, d'un cadre de réflexion commun à l'ensemble des disciplines scolaires, il nous faut cependant mettre en évidence quelques caractéristiques des SES qui conferent une certaine spécificité au processus de transposition didactique dans cette discipline scolaire. 


\section{La transposition didactique: des traits caracté- ristiques spécifiques en Sciences économiques et sociales}

\section{La question de la dépersonnalisation et de la "déformation du savoir»}

Dans le processus de transposition didactique décrit par Chevallard (1985/1991), on assiste à une certaine dépersonnalisation du savoir et le savoir savant serait ainsi séparé de la personne de son producteur. S'il apparaît en effet clair, dans l'ensemble des disciplines scolaires, qu'un savoir doit être détaché de l'espace de production privé du chercheur pour acquérir un statut "public», la question de la dépersonnalisation en SES mérite cependant qu'on s'y arrête.

En mathématiques, nous dit Chevallard, le nom de Fréchet n’apparaît pas lorsqu'on introduit la notion de «distance» au collège. En outre, ce concept, lorsqu'il devient objet d'enseignement, n'est plus utilisé dans la même «sphère mathématique» que le concept créé par le mathématicien, puisqu'on passe de l'analyse (naissance du concept dans la sphère «savante») à la géométrie (introduction du concept en classe de 4ème en 1971) 7 .

En SES, les choses s'avèrent un peu plus complexes que ce qui se passe dans le modèle classique de la transposition didactique. En effet, la quasi-totalité des manuels scolaires actuels accorde une place significative à la présentation des courants théoriques, même si les programmes restent encore prudents quant à la dimension théorique de l'enseignement des SES. Et lorsque cette présentation est faite, compte tenu du caractère pluriparadigmatique de la discipline, elle l'est en référence à des auteurs ou à des courants représentés par des auteurs.

Sur le thème du chômage par exemple, les manuels de terminale actuels présentent, pour la plupart, un certain nombre de théories explicatives du chômage. On parlera ainsi de la «théorie keynésienne», ou de la "théorie du déséquilibre» en citant des économistes comme Malinvaud (1983) ou Benassy (1976). Si certains manuels évoquent la notion de «taux de chômage naturel», ils le font de même en référence à Friedman (1968) qui a introduit ce concept dans la théorie économique. De nombreux manuels présentent également les choix de politiques conjoncturelles qui ont pu être faits par certains gouvernements dans les pays européens des années 1970-1980. Il s'agit alors d'étudier le «dilemme» inflation / chômage. Les déterminants de ce choix sont généralement présentés en référence à Phillips (1958) et à la relation qu'il a mise en évidence entre le taux de croissance des salaires nominaux et le taux de chômage.

En outre, la présentation de ces concepts et des auteurs dans les manuels récents de SES, ne débouche pas, contrairement à ce qui se passe en mathématiques ou dans les sciences de la nature, sur une «déformation» du savoir. On ne change pas de «sphère économique»: le concept de taux de chômage naturel a été créé par Friedman pour expliquer le chômage et pour montrer la supériorité des mécanismes de marché dans la lutte contre les déséquilibres économiques. Il n’a 
pas été créé pour expliquer un autre phénomène économique que celui-là même qui est présenté aux élèves dans les manuels du secondaire. De la même façon, la théorie de l'équilibre de sous-emploi est présentée en référence à Keynes (1936), en précisant qu'il a bâti sa théorie du chômage involontaire dans les années trente, après la crise de 1929. Les élèves travaillent ainsi directement sur les textes des auteurs producteurs du savoir savant.

Chevallard (1985/1991) précise également que dépersonnalisation signifie «objectivation du savoir»: "la textualisation réalise [...] la dissociation entre la pensée en tant que portée par une subjectivité, et ses productions discursives: le sujet est expulsé hors de ses productions» (p. 61). Dans ce sens, il est certain que la validité de la théorie du chômage naturel de Friedman passe par son objectivation, même si le rapport personnel de ce chercheur aux phénomènes économiques et sociaux n'est pas étranger à ses choix théoriques personnels.

Cette référence aux auteurs dans l'enseignement des SES présente cependant un risque de «monumentalisation des savoirs» (Chevallard 2004), les professeurs pouvant être tentés d'exposer les thèses des théoriciens de l'économie et de la sociologie pour elles-mêmes et non pour les schèmes d'intelligibilité du réel qu'elles proposent. Si ce risque de «rapport monumental aux œuvres» est écarté, l'enseignement des SES pourrait utilement être interprété à travers la théorie anthropologique du didactique (Chevallard, 1996). Celle-ci souligne en effet que l'important est de définir les questions auxquelles on se propose de répondre en mobilisant des savoirs validés au sein de communautés scientifiques.

Ainsi, si la transposition didactique en SES peut aisément reprendre à son compte différentes caractéristiques du processus décrit par Chevallard: désyncrétisation, publicité, programmabilité, contrôle social des apprentissages, les choses sont plus complexes en ce qui concerne la dépersonnalisation du savoir. On ne peut pas parler de dépersonnalisation au sens où les concepts et théories seraient enseignés sans référence à leurs auteurs. Les élèves travaillent sur des textes d'économistes et de sociologues, et lorsque ces références sont mobilisées, elles le sont bien dans le champ d'application théorique qui est le leur: il n'y a donc pas en ce sens de "déformation du savoir». Il y a cependant une certaine objectivation du savoir en Sciences économiques et sociales, au sens où le savoir qui est donné à enseigner ne l'est effectivement que lorsqu'il a déjà - depuis longtemps et souvent - été validé par une communauté scientifique, ce qui est bien le propre de la transposition didactique.

\section{Outils d'évaluation et contenus d'enseignement en Sciences économiques et sociales}

La théorie de la transposition didactique considère généralement que les programmes, une fois conçus, sont réinterprétés par les professeurs, à l'aide des manuels notamment, et que l'évaluation externe que constitue l'épreuve du baccalauréat se contente d'apprécier dans quelle mesure chaque élève donne des signes d'adéquation au rapport officiel aux objets d'enseignement. En SES, comme 
dans toutes les disciplines, un contrat didactique spécifique est mis en place en classe de terminale, contrat surdéterminé par l'évaluation au baccalauréat. Le contrat institutionnel explicite, traiter le programme officiel, est souvent modifié par l'exigence prioritaire de préparation des élèves aux épreuves du bac. Entre les programmes et ce qui est effectivement enseigné, il y a un long parcours, dans lequel les instruments d'évaluation jouent un rôle important.

Si l'on prend une nouvelle fois le thème du chômage, on constate par exemple qu'il existe un décalage certain entre les programmes et instructions officielles d'une part et les sujets de baccalauréat proposés aux élèves d'autre part. Même si cet écart tend à se réduire compte tenu des évolutions de la discipline, il faut encore aujourd'hui faire plus, ou autre chose, que ce qu'indiquent les programmes. Le refoulement des interrogations théoriques resurgit dans les sujets de l'examen final constitué par le baccalauréat, révélant ainsi, dans une certaine mesure, un curriculum réel différent du curriculum formel.

Deux conclusions principales ont été dégagées par notre étude des sujets de bac concernant le chômage (Dollo, 2001b):

- Les sujets intègrent beaucoup plus fortement et rapidement que les programmes officiels les débats (sociaux et scientifiques) relatifs à l'emploi et au chômage. L'existence de ces sujets a un effet en retour sur l'enseignement (perceptible notamment à travers le contenu des manuels).

- Alors que les programmes de l'époque ne font que faiblement référence aux approches théoriques, celles-ci sont présentes - explicitement ou implicitement - dans les sujets, ce qui conduit à leur prise en compte dans les enseignements.

Selon nous, cette avance des sujets de bac sur les programmes est la manifestation de la gestion d'une contradiction entre deux objectifs du "projet fondateur»: permettre aux élèves d'accéder à l'intelligence des économies et sociétés contemporaines d'une part, et d'autre part partir des préoccupations des élèves en négligeant la théorie parce que cela pourrait "durcir prématurément» de jeunes esprits. Les sujets de bac trouvent alors la seule solution pour surmonter cette contradiction, à savoir introduire des éléments théoriques pour comprendre les enjeux nouveaux même quand ils ne figurent pas dans les programmes. Car en effet, on ne peut accéder à la compréhension du phénomène du chômage, si on en néglige les explications théoriques.

La confection de sujets de bacs (bien que pilotée par l'Inspection) ne présente pas le même enjeu que la rédaction de programmes officiels. Le programme étant ce qu'il est, on accepte des sujets introduisant des éléments théoriques dès lors qu'ils correspondent à l'objectif "d'intelligence des sociétés contemporaines». Des sujets purement théoriques ne seraient pas acceptés par les commissions de choix des sujets. Mais, dans la mesure où on traite un problème comme le chômage et qu'on ne peut pas le faire sans référence aux analyses théoriques, un compromis est passé: dans les programmes et les instructions officielles, on continue à réaffirmer le "projet fondateur» avec plus ou moins de force (et avec 
une nette évolution dans la période récente). Mais les difficultés de mise en œuvre de ce projet fondateur conduisent à l'introduction, au niveau des sujets de bac notamment (et donc au niveau des pratiques enseignantes elles-mêmes), d'éléments de réflexion théorique et de modélisation des phénomènes économiques et sociaux.

Dans ce cadre, le processus classique de la transposition didactique semble quelque peu bousculé au sens où les pratiques de classes et les sujets proposés aux élèves accélèrent le processus de transposition traditionnellement initié par la noosphère. Les savoirs académiques et théoriques, ceux de la science en train de se faire, sont ainsi parfois freinés par l'institution et les défenseurs du "projet fondateur», mais ils parviennent aux élèves de façon détournée et par une petite porte: les manuels et les professeurs qui ne peuvent éviter d'y recourir pour aborder avec leurs classes les problèmes économiques et sociaux fondamentaux.

\section{Conclusion}

La place de la référence aux savoirs scientifiques en SES fait généralement l'objet d'un débat assez polémique. Une position souvent avancée peut se résumer en disant que l'enseignement des SES ne doit pas être un simple décalque des savoirs universitaires. Sous cette forme-là, on ne peut qu'être d'accord avec cette proposition, mais ce n'est pas alors un problème qui est spécifique aux SES.

En revanche, nous avons pu montrer dans cet article que le concept de transposition didactique était pertinent pour les SES et que les contenus enseignés dans cette discipline se référaient bien à des savoirs produits par des communautés et validés par des communautés savantes. En ce sens, les SES ne sont pas - ou plus - une «anomalie didactique» (Chevallard, 1997). Pas plus que les autres disciplines, les SES ne peuvent prétendre définir de façon purement endogène les contenus enseignés.

Cependant, il existe une spécificité didactique des Sciences économiques et sociales qui tient à deux phénomènes principaux:

- Dans la mesure où il s'agit de conduire les élèves à la compréhension des grands enjeux économiques et sociaux contemporains, les professeurs de SES sont très souvent conduits à adopter une démarche consistant à partir de questions "ombilicales» au sens de Chevallard et à organiser des parcours d'étude et de recherche des élèves afin de mobiliser les savoirs permettant de répondre à ces questions. En ce sens, les SES se révèlent assez proches, en dépit de certaines dénégations, des formulations les plus récentes de la théorie de la transposition didactique.

- Dans la mesure où les savoirs de référence sont pluriparadigmatiques, la dépersonnalisation prend une forme spécifique en SES, articulant à la fois une objectivation des savoirs et un rattachement des concepts et des théories aux auteurs et aux courants qui les ont produits. 


\section{Notes}

Je tiens à remercier Gérard Sensevy pour sa lecture critique attentive et ses précieux conseils. Vifs remerciements également aux lecteurs anonymes du comité de lecture de la Revue pour les commentaires instructifs et éclairants qu'ils ont fournis sur la version initiale de ce texte, même si les propos tenus n'engagent évidemment que son auteure.

1 Selon une expression de Bremond et Lanta (1995).

2 Marcel Roncayolo est un géographe à qui le ministère de l'Education nationale confie (ainsi qu'à Guy Palmade, historien de l'économie) en 1965 la charge de définir ce que pourrait être une Initiation aux faits économiques et sociaux destinée à des élèves de l'enseignement général.

3 Pour une histoire détaillée et exhaustive de l'utilisation du concept de transposition didactique en mathématiques, on se reportera utilement à Mercier (2002).

4 Le qualificatif de «savant» a souvent porté à controverses dans la théorie de la transposition didactique. Mais dans les développements de cette théorie, le qualificatif de savant n'est plus essentiel. Il peut ainsi exister un - ou plusieurs - savoir(s) de référence qui, dans certains cas, peuvent se révéler savants au sens académique du terme.

5 Nous ne travaillons dans ce texte que sur les aspects «externes» de la transposition didactique. Des recherches en cours tendent à étudier les situations didactiques auxquelles sont confrontés les élèves, pour analyser la façon dont elles donnent du sens aux savoirs enseignés.

6 A titre d'anecdote, je peux relater le fait que lorsque j'étais une jeune professeure dans le second degré, un Inspecteur m'avait dit lors de l'entretien consécutif à une inspection: «si vous souhaitez que les élèves comprennent vraiment ce qu'est la monnaie, alors videz vos poches sur la table... Que verront-ils? Quelques billets, des pièces, un carnet de chèque... Là ils palperont vraiment la monnaie du bout des doigts».

7 On constate cependant une évolution récente dans les programmes de lycée: les professeurs sont invités à introduire des éléments d'histoire des sciences et des mathématiques. Par exemple, dans le manuel de terminale $S$ de la collection "Déclic» paru chez Hachette en 1998, on trouve, çà et là, quelques allusions à des mathématiciens célèbres. Ainsi, dans le chapitre sur les logarithmes népériens, une petite introduction historique cite des mathématiciens du XVIIème siècle et précise que les logarithmes ont notamment été introduits pour résoudre les problèmes posés par la lourdeur des calculs en astronomie.

\section{Bibliographie}

Bachelard, G. (1983). La formation de l'esprit scientifique [original publié 1938]. Paris: Librairie Philosophique J. Vrin

Beitone, A., Decugis, M.-A., Dollo, C. \& Rodrigues, C. (2004). Les Sciences économiques et sociales, enseignement et apprentissages. Bruxelles: De Boeck

Benassy, J.-P. (1976). Macroéconomie et théorie du déséquilibre. Paris: Dunod.

Bremond, J. \& Lanta, H. (1995). La pédagogie des Sciences économiques et sociales: mythe fondateur ou réalité? In P. Combemale (Coord.), Les Sciences économiques et sociales (pp. 47-71). Paris: Hachette Education.

Chatel, E. (1995a). Transformation des savoirs en Sciences économiques et sociales, Revue Française de Pédagogie, 112, 9-20.

Chatel, E. (1995b). Insertion institutionnelle et enjeux didactiques. In P. Combemale (Coord.), Les Sciences économiques et sociales (pp. 7-36). Paris: Hachette Education. 
Chatel, E. (1999). Légitimité savante et valeur scientifique dans l'enseignement des SES. Une approche critique du concept de transposition didactique. Documents pour l'enseignement économique et social, 116, 23-28.

Chatel, E., Caron, P., Fenet-Chalaye, C., Le Merrer, P., Pasquier, P. \& Simula, L. (1993). Enseigner les Sciences Economiques et Sociales, introduction à une réflexion didactique (2ème édition). [1ère édition 1990]. Paris: INRP.

Chatel, E., Caron, P., Le Merrer, P., Pasquier, P., Richet, A.. \& Simula, L. (1995). Marchés et prix. Savoirs enseignés et façons d'enseigner en Sciences économiques et sociales. Paris: INRP.

Chevallard, Y. (1985). La transposition didactique, du savoir savant au savoir enseigné [Réédition augmentée 1991]. Grenoble: La Pensée Sauvage.

Chevallard, Y. (1994). Les processus de transposition didactique et leur théorisation. In G. Arsac, Y. Chevallard, J.-L. Martinand, \& A. Tiberghien (Coord.), La transposition didactique à l'épreuve (pp. 135-180). Grenoble: La Pensée Sauvage.

Chevallard, Y. (1996). Concepts fondamentaux de la didactique: perspectives apportées par une approche anthropologique. In J. Brun (Dir.), Didactique des mathématiques (pp. 145196). [Republié de Recherches en Didactique des Mathématiques, 12 (1), 73-112, 1992]. Lausanne: Delachaux \& Niestlé.

Chevallard, Y. (1997). L'enseignement des SES est-il une anomalie didactique? Skholê, 6, 25-37.

Chevallard, Y. (2004). La place des mathématiques vivantes dans l'éducation secondaire: transposition didactique des mathématiques et nouvelle épistémologie scolaire. 3e Université d'été Animath [Page Web]. Accès: [http://www.animath.fr/UE/UE04/chevallard.pdf].

Combemale, P. (1995). Une discipline indisciplinée. In P. Combemale (Coord.), Les Sciences économiques et sociales (pp. 5-6). Paris: Hachette Education

Dollo, C. (2001a). Quels déterminants pour l'évolution des savoirs scolaires en Sciences Economiques et Sociales? (l'exemple du chômage). Thèse de Sciences de l'Education, Université de Provence, CIRADE, Aix en Provence [Page Web]. Accès: [http://www.aix-mrs.iufm.frlformations/filieres/ses/didactiquelthesecd.pdf].

Dollo, C. (2001b). Outils d'évaluation et contenus de formation: analyse des sujets de bac en Sciences Economiques et Sociales (l'exemple du thème du chômage en classe de Terminale). Actes du 14ème colloque international de l'Admee Europe, Les interactions entre formation et évaluation, Aix en Provence, 11-13 janvier 2001.

Dollo, C. \& Johsua, S. (2002). Conceptions d'élèves et diversité des paradigmes en Sciences économiques et sociales (l'exemple du chômage). L'Année de la recherche en Sciences de l'Education, 1/1, 181-208.

Dollo, C. \& Parayre, S. (2005). Et l'amour dans tout ça? Des conceptions des élèves à la construction de savoirs scientifiques sur la famille. Skholê, hors série 1, 39-51.

Friedman, M. (1968). The role of Monetary Policy. American Economic Review, 58, 1-17.

Hadjian, J. (1994). Continuité ou discontinuité ... de l'enseignement des SES en lycée? Documents pour l'enseignement économique et social, 96, 75-79.

Johsua, S. (1998). Des savoirs et de leur étude: vers un cadre de réflexion pour l'approche didactique. L'Année de la Recherche en éducation, 1/1, 79-97.

Keynes, J.-M. (1969). Théorie générale de l'emploi, de l'intérêt et de la monnaie [Original publié 1936]. Paris: Payot.

Legardez, A. (2001). La didactique des sciences économiques et sociales, bilan et perspectives. Aix en Provence: Presses de l'Université de Provence.

Malinvaud, E. (1983). Essais sur la théorie du chômage. Paris: Calmann-Lévy.

Mercier, A. (2002). La transposition des objets d'enseignement et la définition de l'espace didactique, en mathématiques. Revue française de pédagogie, 141, 135-171.

Mercier, A., Schubauer-Leoni, M.-L. \& Sensevy, G. (2002). Vers une didactique comparée. Revue française de pédagogie, 141, 5-16. 
Phillips, A. W. (1958) The relation between unemployment and the rage of change of money wage rates in the United Kingdom, 1861-1957. Economica, 25, 283-299.

Sensevy, G., Mercier, A. \& Schubauer-Leoni, M.-L. (2000). Vers un modèle de l'action didactique du professeur à propos de la course à 20. Recherches en Didactique des Mathématiques, 20, (3), 263-304.

Triby, E. (1995). Les Sciences économiques et sociales sont-elles une discipline? L'interrogation de la didactique. In P. Combemale (Coord.), Les sciences économiques et sociales (pp. 113-128). Paris: Hachette Education.

Verret, M. (1975). Le temps des études. Paris: Librairie Honoré Champion.

Mots clés: Transposition didactique, didactique des Sciences économiques et sociales, didactique comparée

\section{Generischkeit / Eigentümlichkeit eines Begriffs: die didaktische Transposition in den Wirtschafts- und Sozialwissenschaften}

\section{Zusammenfassung}

Der Gründungsentwurf der Wirtschafts- und Sozialwissenschaften schien am Anfang unvereinbar mit dem Anspruch, dass die Didaktik des Fachs die Theorie der didaktischen Transposition berücksichtigen sollte. Absicht dieses Artikels ist es deshalb, die heuristische Bedeutung des für die Wirtschafts- und Sozialwissenschaften von Verret eingeführten und dann von Chevallard theoretisierten Begriffs anschaulich zu machen.

Selbst wenn dieser anfangs in der Didaktik der Mathematik oder der Physik benutzte Begriff auch in den Wirtschafts- und Sozialwissenschaften völlig operativ war und ist, ist es möglich, bestimmte Spezifizitäten der didaktischen Transposition im Bereich der Wirtschafts- und Sozialwissenschaften anschaulich zu machen. Diese Spezifizitäten sind mit den Eigentümlichkeiten dieses Schulfachs verbunden. Dieser Artikel ist in eine komparistitische Perspektive einzureihen

Schlagworte: didaktische Transposition, Didaktik der Wirtschafts- und Sozialwissenschaften, vergleichende Didaktik

\section{Genericità / specificità di un concetto: La trasposizione didattica nelle Scienze economiche e sociali}

Riassunto

Inizialmente il "progetto fondatore» delle Scienze economiche e sociali (SES) sembrava incompatibile con la presa in considerazione, da parte della didattica della disciplina, della teoria della trasposizione didattica. L'obiettivo di questo articolo è tuttavia di mettere in evidenza la portata euristica per le SES del concetto inaugurato da Verret e poi teorizzato da Chevallard. 
Ma si noterà che, anche se il concetto utilizzato inizialmente in didattica della matematica o delle scienze fisiche è perfettamente operatorio per le SES, è possibile mettere in evidenza certi tratti specifici della trasposizione didattica nelle SES, tenendo presente le caratteristiche inerenti a questa disciplina scolastica. Questo lavoro s'inserisce quindi in una prospettiva comparata.

Parole chiave: Trasposizione didattica, didattica delle Scienze economiche e sociali, didattica comparata

\section{Genericity /Specificity of a concept: Didactic transposition in Economics and Social Sciences}

\section{Abstract}

The Economics and Social Sciences (ESS) "founding base" seemed at first incompatible with the fact that the theory of didactic transposition could be taken into account by the didactic of ESS. This article aims at bringing to the fore, for ESS, the heuristic scope of the concept originated by Verret which was later theorized by Chevallard. Yet, one will notice that, even if this concept was originally used in Mathematics or Physics didactic and is perfectly operating for ESS, it is possible to bring to the fore specific patterns of didactic transposition in ESS linked with the own features of this school subject. Therefore, this paper comes within a comparative angle.

Key words: didactic transposition, didactic of Economics and Social Sciences, comparative didactic 